\title{
Transductive Auxiliary Task Self-Training for Neural Multi-Task Models
}

\author{
Johannes Bjerva ${ }^{1}$, Katharina Kann ${ }^{2}$, Isabelle Augenstein ${ }^{1}$ \\ ${ }^{1}$ Department of Computer Science, University of Copenhagen, Denmark \\ ${ }^{2}$ Center for Data Science, New York University, USA \\ bjerva, augenstein@di.ku.dk, kann@nyu.edu
}

\begin{abstract}
Multi-task learning and self-training are two common ways to improve a machine learning model's performance in settings with limited training data. Drawing heavily on ideas from those two approaches, we suggest transductive auxiliary task self-training: training a multi-task model on (i) a combination of main and auxiliary task training data, and (ii) test instances with auxiliary task labels which a single-task version of the model has previously generated. We perform extensive experiments on 86 combinations of languages and tasks. Our results are that, on average, transductive auxiliary task self-training improves absolute accuracy by up to $9.56 \%$ over the pure multitask model for dependency relation tagging and by up to $13.03 \%$ for semantic tagging.
\end{abstract}

\section{Introduction}

When data for certain tasks or languages is not readily available, different approaches exist to leverage other resources for the training of machine learning models. Those are commonly either instances from a related task or unlabelled data: During multi-task training (Caruana, 1993), a model learns from examples of multiple related tasks at the same time and can therefore benefit from a larger overall number of training instances. Self-training (Yarowsky, 1995; Riloff et al., 2003), in contrast, denotes the process of iteratively training a model, using it to label new examples, and adding the most confident ones to the training set before repeating the training. As data without gold standard annotations is used, self-training can be considered a special case of semi-supervised training.

In this work, we propose transductive auxiliary task self-training, based on a combination of multi-task training and self-training: We use the available auxiliary task data to obtain a high-performing single-task model for the auxiliary task, which we then use to label the main task test set with auxiliary task labels. Subsequently, we train a multi-task model on both tasks, while including instances with the newly generated silver standard auxiliary task labels.

Transductive auxiliary task self-training is an extremely cheap procedure, requiring only small amounts of additional computing time, compared to the obvious alternative of manually producing more labels. Our approach is transductive since the model generalises from specific training examples to specific test examples. In particular, training on auxiliary task labels for the test set, which have been produced by the single-task model, yields a final multi-task model, which satisfies the defining criterion of transductive inference that predictions depend on the test data (Vapnik, 1998). Note that we do not require gold standard test labels for either task.

In addition to presenting our method, we investigate three research questions $(\mathbf{R Q s})$ :

RQ 1: For which tasks and dataset sizes does transductive auxiliary task self-training help most?

RQ 2: Can a model trained with our cost-free transductive auxiliary task self-training perform similarly to or better than a model trained on additional manual annotations for the auxiliary task?

RQ 3: Even without considering reduced costs, are there scenarios where it is better to perform transductive auxiliary task self-training than adding more main task examples?

In order to find generalisable answers to these research questions, we experiment with several tasks, languages and numbers of training samples. We consider the low-level auxiliary task of part-of-speech tagging and two main tasks: dependency relation (DepRel) tagging and semantic tagging. We furthermore compare with an unsuper- 


\begin{tabular}{|c|c|c|c|c|c|c|c|c|c|c|c|c|c|}
\hline We & must & draw & ttention & to & the & distribution & of & this & form & in & those & dialects & Sentence \\
\hline PRON & AUX & VERB & NOUN & $A D P$ & DET & NOUN & $A D P$ & DET & NOUN & $\mathrm{ADP}$ & DET & NOUN & POS \\
\hline nsubj & aux & root & obj & case & $\operatorname{det}$ & obl & case & $\operatorname{det}$ & nmod & case & $\operatorname{det}$ & obl & DepRel \\
\hline PRO & $\mathrm{NEC}$ & EXS & $\mathrm{CON}$ & REL & $\mathrm{DEF}$ & $\mathrm{CON}$ & AND & PRX & $\mathrm{CON}$ & REL & DST & $\mathrm{CON}$ & SemTag \\
\hline
\end{tabular}

Figure 1: POS tags, DepRel labels and semantic tags for an example sentence.

vised auxiliary task baseline, to show that our results are not simply a result of domain adaptation effects. We experiment on 41 languages, yielding a total of 86 unique language-task combinations. We find that, on average, transductive auxiliary task self-training improves absolute accuracy by up to $9.56 \%$ and $13.03 \%$ over the pure multi-task model for DepRel tagging and semantic tagging, respectively.

\section{Neural Sequence Labelling}

\subsection{Tasks}

Figure 1 shows a sentence with annotations for the three linguistic tasks considered in this paper, which we will describe in the following. ${ }^{1}$

Part-of-speech (POS) tagging is the task of assigning morpho-syntactic tags to each word in a sentence. We use it as an auxiliary task, since respective datasets are available for many languages. It is also a relatively easy task, with state-of-theart models typically achieving over $95 \%$ accuracy (Plank et al., 2016). We use the Universal Dependencies (UD) POS tag set (Nivre et al., 2016).

Dependency relation (DepRel) labelling is the task of assigning dependency labels to each word in a sentence. In our experiments, we use the Universal Dependencies labels (Nivre et al., 2016). We use this task as a main task. Both this task and POS tagging are morpho-syntactic tasks.

Semantic Tagging (SemTag) is the task of assigning a semantic tag to each word in a sentence. We use the labels from the Parallel Meaning Bank (PMB, Abzianidze et al. (2017); Bjerva et al. (2016)). This tag set was designed for multilingual semantic parsing and, therefore, to generalise across languages. As this task is relatively challenging, we use it as a main task. While the UD data is available for 41 languages, the PMB data is only available for four (English, Italian, Dutch, and German).

FreqBin is the task of predicting the binned frequency of a word, as introduced by Plank et al.

\footnotetext{
${ }^{1}$ The example is taken from PMB document 01/3421, which has gold standard SemTags. The UD POS and DepRel tags were obtained using UD-Pipe (Straka et al., 2016).
}

(2016). We use this task as an unsupervised auxiliary baseline, with frequencies obtained from our training data.

\subsection{Model Architecture}

We approach sequence labelling by using a variant of a bidirectional recurrent neural network, which uses both preceding and succeeding context when predicting the label of a word. This choice was made as such models at the same time obtain high performance on all three tasks and lend themselves nicely to multi-task training via hard parameter sharing. This system is based on the hierarchical bi-LSTM of Plank et al. (2016) and is implemented using DyNet (Neubig et al., 2017). On the subword-level, the LSTM is bi-directional and operates on characters (Ballesteros et al., 2015; Ling et al., 2015). Second, a context bi-LSTM operates on the word level, from which output is passed on to a classification layer.

Multi-task training is approached using hard parameter sharing (Caruana, 1993). We consider $T$ datasets, each containing pairs of input-output sequences $\left(w_{1: n}, y_{1: n}^{t}\right), w_{i} \in V, y_{i}^{t} \in L^{t}$. The input vocabulary $V$ is shared across tasks, but the outputs (tagsets) $L^{t}$ are task dependent. At each step in the training process we choose a random task $t$, followed by a randomly chosen batch of training instance. Each task is associated with an independent classification function, but all tasks share the hidden layers. We train using the Adam optimisation algorithm (Kingma and $\mathrm{Ba}, 2014$ ) over a maximum of 10 epochs together with early stopping.

\section{Transductive Auxiliary Task Self-Training}

Manual annotation of data for main or auxiliary tasks is time-consuming and expensive. Instead, we propose to use a preliminary single-task model to label the main task test data with auxiliary task labels which can then be leveraged to train an improved multi-task model.

Transductive auxiliary task self-training is based on two main ideas. First, we assume that the auxiliary task is easier than the main task, such 
that a high performance can be achieved on it. Hence, the model will be confident about the auxiliary task labels, as is required for self-training. Second, we choose a transductive approach, because we assume that not all auxiliary task examples will lead to equal improvements on the main task. In particular, we expect auxiliary task labels for the test instances to be most useful, since information about those instances is most relevant for the prediction of the main task labels on this data. Similarly to contextualised word representations, this offers an additional signal for the test set instances, as we obtain this through predicted auxiliary labels rather than direct encoding of the context (Devlin et al., 2018; Peters et al., 2018).

\subsection{Algorithm}

Our proposed algorithm is shown in Algorithm 1. We start by first training a single-task model on the available auxiliary task training data, which then predicts labels for the raw input sentences from the main task test set. Note that we neither observe nor require any labels for this test set, neither for the auxiliary nor for the main task. The labels which the preliminary single-task model predicts are then added to the train set of the auxiliary task for training of the final multi-task model.

Although a transductive approach requires training a new model for each test set, sequencelabelling models such as bi-LSTMs are usually quick to train even on single CPUs, with a full self-training iteration in this paper completing in a matter of hours.

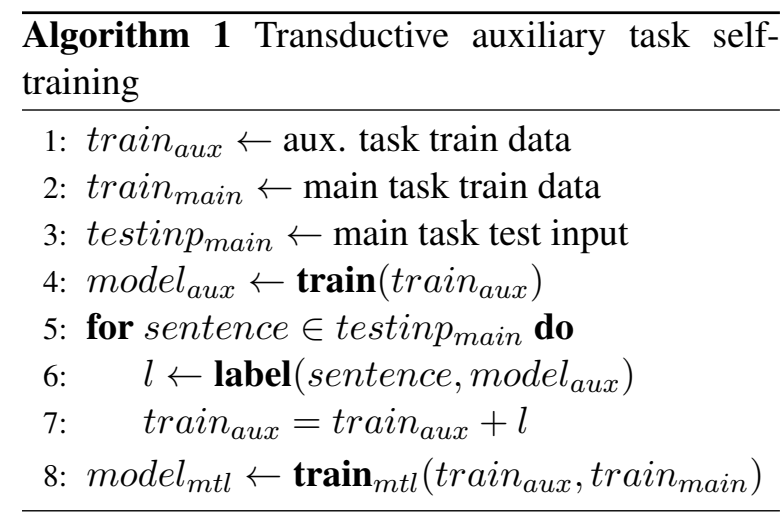

\section{Experiments}

The experiments described in this section aim at answering the research questions raised in $\S 1$, concerned with the best settings for transductive auxiliary task self-training, as well as the theoretical question how it compares to adding additional (expensive) gold-standard annotations for the main and the auxiliary tasks. To ensure that our findings are generalisable, we use a large sample of 56 treebanks, covering 41 languages and several domains. Although this experimental set-up would allow us to run multilingual experiments, we only train monolingual models, and aggregate results across languages and treebanks. We investigate three tasks; two of them being morpho-syntactic (POS tagging and DepRel tagging) and one being semantic (semantic tagging). In all cases, POS is the auxiliary task, and either POS tagging or DepRel tagging is the main task. Experiments are run in several low-resource settings, varying the amount of main task data.

We run experiments under four conditions, in addition to using an MTL baseline. We compare (i) adding gold standard test annotations for the auxiliary task only (Aux-ST ceiling), (ii) transductive auxiliary task self-training, as described in Algorithm 1 (Aux-ST), (iii) adding gold standard train annotations for the auxiliary task only (Extra Aux), or (iv) adding gold standard train annotations for the main task only (Extra Main). We expect (iii) and (iv) to constitute challenging conditions to beat, as we are in effect giving our model more annotated data, which is normally expensive to come by.

\subsection{Data}

We run experiments on the task-combinations DepRel-POS and Semtag-POS for all available languages and datasets. Additionally, we reduce our training sets to $10 \mathrm{k}, 1 \mathrm{k}, 0.5 \mathrm{k}$, and $0.1 \mathrm{k}$ sentences in order to investigate various low-resource scenarios. For semantic tagging, the 10k setting is omitted as we do not have enough training data.

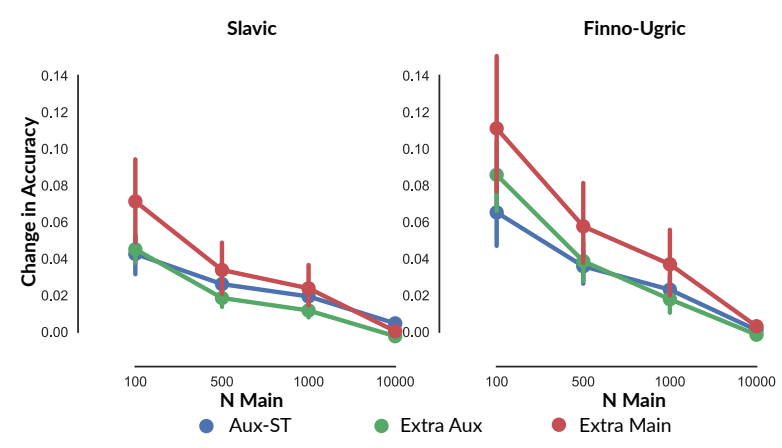

Figure 2: Results for UD treebanks. 


\begin{tabular}{rrr|rrr}
\hline N Main & MTL & Aux-ST ceiling & Aux-ST & Extra Aux & Extra Main \\
\hline $10 \mathrm{k}$ & 86.39 & $* 3.79 \%$ & $* \mathbf{1 . 9 7 \%}$ & $-0.30 \%$ & $0.19 \%$ \\
$1 \mathrm{k}$ & 79.19 & $* 7.42 \%$ & $* 5.39 \%$ & $* 1.74 \%$ & $* \mathbf{7 . 0 1 \%}$ \\
$0.5 \mathrm{k}$ & 75.77 & $* 8.69 \%$ & $* 6.85 \%$ & $* 2.64 \%$ & $* \mathbf{1 0 . 1 7 \%}$ \\
$0.1 \mathrm{k}$ & 66.31 & $* 11.32 \%$ & $* 9.56 \%$ & $* 4.97 \%$ & $* \mathbf{1 8 . 1 5 \%}$ \\
\hline $1 \mathrm{k}$ & 67.82 & $\mathrm{n} / \mathrm{a}$ & $* \mathbf{1 . 7 4 \%}$ & $0.05 \%$ & $0.64 \%$ \\
$0.5 \mathrm{k}$ & 63.32 & $\mathrm{n} / \mathrm{a}$ & $* \mathbf{4 . 6 0 \%}$ & $0.83 \%$ & $* 2.31 \%$ \\
$0.1 \mathrm{k}$ & 50.44 & $\mathrm{n} / \mathrm{a}$ & $* \mathbf{1 3 . 0 3} \%$ & $* 4.93 \%$ & $* 11.58 \%$ \\
\hline
\end{tabular}

Table 1: Macro-averaged changes in accuracy from the MTL baseline for DepRel - POS (top), SemTag - POS (bottom). We compare adding gold standard test annotations for the aux task (Aux-ST ceiling), transductive aux task self-training (Aux-ST), adding gold standard train annotations for the aux task (Extra Aux), or for the main task (Extra Main) randomly. Significant $(p<0.05)$ differences from the baseline are marked with *.

\subsection{Results and Discussion}

Table 1 contains results of the experiments macroaveraged across all languages and treebanks in the UD and across all languages in the PMB. Figure 2 contains results for two typologically distinct language families, Slavic and Finno-Ugric.

Across all data sizes, self-training on the auxiliary task is significantly better than the baseline multi-task model without self-training. The results on DepRel tagging show that, when the main task data is sufficiently large, it is more beneficial to do transductive auxiliary task self-training than it is to further increase the size of the main dataset. For semantic tagging, we find this to hold for all of our training data size settings. Our comparison with the FreqBin task does not yield substantial improvements, with mean differences compared to standard MTL at $-0.001 \%$ (stdev. 0.022).

To rule out that any gains in the self-training conditions are not due to increased vocabulary, we ran experiments with pre-trained word embeddings which included the raw text from the test set and found no significant differences. This can be explained by the fact that, although out-ofvocabulary rate is reduced to zero in this condition, the test set is still relatively small. Thus, the word embeddings do not have much distributional information with which to arrive at good word representations for previously out-of-vocabulary words.

In RQ1, we asked for which task and dataset sizes transductive auxiliary task self-training is most beneficial. We found benefits across the board, with larger effects when the main task training set is small.
In RQ2, we asked whether using transductive auxiliary task self-training might even be better than the costly process of manually expanding the data with gold standard auxiliary data for random samples. We found that this depends on the main task and the size of its training set. For DepRels, with a low amount of main task data, the largest increase in accuracy is found by adding more main task data. However, given sufficient main task data, adding highly relevant auxiliary task samples, even ones which are potentially erroneous, is more beneficial. In the case of semantic tagging, however, transductive auxiliary task self-training is always more beneficial. As expected, the usefulness of self-training as well as adding extra auxiliary or main task data decreases with increasing dataset size.

In RQ3, we asked whether there are cases in which using auxiliary task data is preferable to annotating and adding more main task samples. We found that this is the case when using our proposed method of transductive auxiliary task self-training for all training set sizes for semantic tagging, and in the 10k setting for DepRel tagging.

\section{Related Work}

Self-training has been shown to be a successful learning approach (Nigam and Ghani, 2000), e.g., for word sense disambiguation (Yarowsky, 1995) or AMR parsing (Konstas et al., 2017). Samples in self-training are typically selected according to confidence (Zhu, 2005) which requires a proxy to measure it. This can be the confidence of the model (Yarowsky, 1995; Riloff et al., 2003) or the agreement of different models, as used in tri-training (Zhou and Li, 2005). Another option is curriculum learning, where selection is based on learning difficulty, increasing the difficulty during learning (Bengio et al., 2009). In contrast, we build upon the assumption that the auxiliary task examples are ones a model can be certain about.

In multi-task learning, most research focuses on understanding which auxiliary tasks to select, or on how to share between tasks (Søgaard and Goldberg, 2016a; Lin et al., 2019; Ruder and Plank, 2017; Augenstein et al., 2018; Ruder et al., 2019). For instance, Ruder and Plank (2017) find that similarity as well as diversity measures applied to the main vs. auxiliary task datasets as a whole are useful in selecting auxiliary tasks. In the context of sequence labelling, many combinations 
of tasks have been explored (Søgaard and Goldberg, 2016b; Martínez Alonso and Plank, 2017; Bjerva, 2017). Ruder et al. (2019) present a flexible architecture, which learns which parameters to share between a main and an auxiliary task. One of the few examples where multi-task learning is combined with other methods is the semisupervised approach by Chao and Sun (2012), where main task labels are assigned to unlabelled instances which are then added to the main task dataset. However, to the best of our knowledge, no one has applied self-training to label additional instances with auxiliary task labels.

\section{Conclusion}

We introduced transductive auxiliary task selftraining, a straightforward way to improve the performance of multi-task models. Concretely, we applied the idea of self-training to auxiliary tasks, in order to automatically label the main task test data with auxiliary task labels which we subsequently included into the training set for multi-task learning. In experiments on 41 different languages we obtained improvements of up to $9.56 \%$ absolute accuracy over the pure multi-task model for DepRel tagging and up to $13.03 \%$ absolute accuracy for semantic tagging. We further showed that transductive auxiliary task self-training is more effective than randomly choosing additional gold standard auxiliary task data. In some settings, in addition to not needing additional annotation, it even led to a better performing model than adding a comparable amount of extra gold standard main task data.

\section{References}

Lasha Abzianidze, Johannes Bjerva, Kilian Evang, Hessel Haagsma, Rik van Noord, Pierre Ludmann, Duc-Duy Nguyen, and Johan Bos. 2017. The Parallel Meaning Bank: Towards a Multilingual Corpus of Translations Annotated with Compositional Meaning Representations. In Proceedings of EACL.

Isabelle Augenstein, Sebastian Ruder, and Anders Søgaard. 2018. Multi-Task Learning of Pairwise Sequence Classification Tasks over Disparate Label Spaces. In Proceedings of NAACL.

Miguel Ballesteros, Chris Dyer, and Noah A Smith. 2015. Improved Transition-based Parsing by Modeling Characters instead of Words with LSTMs. In Proceedings of EMNLP.
Yoshua Bengio, Jérôme Louradour, Ronan Collobert, and Jason Weston. 2009. Curriculum learning. In Proceedings of ICML.

Johannes Bjerva. 2017. One Model to Rule them all: Multitask and Multilingual Modelling for Lexical Analysis. Ph.D. thesis, University of Groningen.

Johannes Bjerva, Barbara Plank, and Johan Bos. 2016. Semantic Tagging with Deep Residual Networks. In COLING, pages 3531-3541.

Rich Caruana. 1993. Multitask Learning: A Knowledge-Based Source of Inductive Bias. In Proceedings of ICML.

Guoqing Chao and Shiliang Sun. 2012. Semisupervised Multitask Learning via Self-training and Maximum Entropy Discrimination. In Proceedings of International Conference on Neural Information Processing.

Jacob Devlin, Ming-Wei Chang, Kenton Lee, and Kristina Toutanova. 2018. Bert: Pre-training of deep bidirectional transformers for language understanding. arXiv preprint arXiv:1810.04805.

Diederik Kingma and Jimmy Ba. 2014. Adam: A Method for Stochastic Optimization. arXiv preprint arXiv:1412.6980.

Ioannis Konstas, Srinivasan Iyer, Mark Yatskar, Yejin Choi, and Luke Zettlemoyer. 2017. Neural AMR: Sequence-to-Sequence Models for Parsing and Generation. In Proceedings of ACL.

Yu-Hsiang Lin, Chian-Yu Chen, Jean Lee, Zirui Li, Yuyan Zhang, Mengzhou Xia, Shruti Rijhwani, Junxian He, Zhisong Zhang, Xuezhe Ma, et al. 2019. Choosing Transfer Languages for CrossLingual Learning. arXiv:1905.12688.

Wang Ling, Chris Dyer, Alan W. Black, Isabel Trancoso, Ramon Fermandez, Silvio Amir, Luís Marujo, and Tiago Luís. 2015. Finding Function in Form: Compositional Character Models for Open Vocabulary Word Representation. In Proceedings of EMNLP.

Héctor Martínez Alonso and Barbara Plank. 2017. When is multitask learning effective? Semantic sequence prediction under varying data conditions. In EACL.

Graham Neubig, Chris Dyer, Yoav Goldberg, Austin Matthews, Waleed Ammar, Antonios Anastasopoulos, Miguel Ballesteros, David Chiang, Daniel Clothiaux, Trevor Cohn, et al. 2017. Dynet: The dynamic neural network toolkit. arXiv preprint arXiv:1701.03980.

Kamal Nigam and Rayid Ghani. 2000. Analyzing the effectiveness and applicability of co-training. In Proceedings of Information and Knowledge Management. 
Joakim Nivre, Marie-Catherine de Marneffe, Filip Ginter, Yoav Goldberg, Jan Hajic, Christopher D. Manning, Ryan T. McDonald, Slav Petrov, Sampo Pyysalo, Natalia Silveira, Reut Tsarfaty, and Daniel Zeman. 2016. Universal Dependencies v1: A Multilingual Treebank Collection. In Proceedings of LREC.

Matthew Peters, Mark Neumann, Mohit Iyyer, Matt Gardner, Christopher Clark, Kenton Lee, and Luke Zettlemoyer. 2018. Deep Contextualized Word Representations. In NAACL, pages 2227-2237. Association for Computational Linguistics.

Barbara Plank, Anders Søgaard, and Yoav Goldberg. 2016. Multilingual Part-of-Speech Tagging with Bidirectional Long Short-Term Memory Models and Auxiliary Loss. In Proceedings of ACL (Short Papers).

Ellen Riloff, Janyce Wiebe, and Theresa Wilson. 2003. Learning Subjective Nouns using Extraction Pattern Bootstrapping. In Proceedings of CoNLL.

Sebastian Ruder, Joachim Bingel, Isabelle Augenstein, and Anders Søgaard. 2019. Latent Multi-task Architecture Learning. In Proceedings of AAAI.

Sebastian Ruder and Barbara Plank. 2017. Learning to select data for transfer learning with Bayesian Optimization. In Proceedings of EMNLP.

Anders Søgaard and Yoav Goldberg. 2016a. Deep multi-task learning with low level tasks supervised at lower layers. In Proceedings of ACL (Short Papers).

Anders Søgaard and Yoav Goldberg. 2016b. Deep multi-task learning with low level tasks supervised at lower layers. In $A C L$.

Milan Straka, Jan Hajic, and Jana Straková. 2016. UDPipe: Trainable pipeline for processing CoNLL-U files performing tokenization, morphological analysis, POS tagging and parsing. In Proceedings of LREC.

Vladimir N Vapnik. 1998. Statistical Learning Theory. John Wiley.

David Yarowsky. 1995. Unsupervised Word-Sense Disambiguation Rivaling Supervised Methods. In Proceedings of ACL.

Zhi-Hua Zhou and Ming Li. 2005. Tri-training: exploiting unlabeled data using three classifiers. IEEE Transactions on Knowledge and Data Engineering, 17:1529-1541.

Xiaojin Zhu. 2005. Semi-Supervised Learning Literature Survey. Technical Report 1530, Computer Sciences, University of Wisconsin-Madison. 